\title{
Attenuated Release of Biologically Active Luteinizing Hormone in Healthy Aging Men
}

Randall J. Urban, ${ }^{\star}$ Johannes D. Veldhuis, ${ }^{*}$ Robert M. Blizzard, ${ }^{\ddagger}$ and Maria L. Dufau ${ }^{5}$

*Division of Endocrinology and Metabolism, Department of Internal Medicine, ${ }^{\ddagger}$ Department of Pediatrics, University of Virginia

School of Medicine, Charlottesville, Virginia 22908; ${ }^{\S}$ Endocrinology and Reproduction Research Branch, The National

Institute of Child Health and Human Development, Bethesda, Maryland 20892

\begin{abstract}
To examine the biological quality and physiologically pulsatile mode of endogenous luteinizing hormone release in active, healthy aging men, we used the rat interstitial-cell testosterone in vitro bioassay to probe $\mathrm{LH}$ bioactivity in response to $(a)$ endogenous gonadotropin-releasing hormone (GnRH) action (basal pulsatile bioactive LH secretion); (b) exogenous GnRH stimulation (10 $\mu \mathrm{g} \mathrm{IV}$ pulses); and (c) inhibition of endogenous estrogen negative feedback (treatment with a nonsteroidal antiestrogen, tamoxifen). Basally, some healthy older men exhibited evidence of neuroendocrine dysfunction, reflected by irregular bursts of bioactive $\mathrm{LH}$ release followed by transiently low plasma bio:immuno (B:I) LH ratios. However, mean basal plasma bioactive LH concentrations, B:I ratios, and spontaneous LH pulse properties (peak frequency, amplitude, duration, and enhanced B:I ratios within LH peaks) were not altered in older men. On the other hand, augmentation of bioactive LH secretion and enhancement of plasma B:I ratios by pulsed injections of exogenous GnRH were either significantly reduced or absent in older men. In addition, although tamoxifen increased bioactive LH pulse frequency in both age groups and facilitated exogenous GnRH action in some subjects, older men increased their 12-h mean bioactive LH concentrations, B:I ratios, and bioactive $\mathrm{LH}$ peak amplitudes to a significantly lesser degree than young men.

In summary, young and older healthy men exhibit similar mean basal plasma bioactive LH concentrations and spontaneous LH pulse properties. However, pituitary bioactive LH reserve is markedly attenuated in older men challenged with either exogenous GnRH or antiestrogen. Accordingly, we conclude that healthy aging men manifest an impaired secretory reserve for biologically active $\mathrm{LH}$ release.
\end{abstract}

\section{Introduction}

The physiological mode of secretion of immunoreactive and biologically active luteinizing hormone has been well-characterized in healthy men under $30 \mathrm{yr}$ of age (1-3). However, few studies have examined the spontaneous pulsatile release of immunoactive LH in healthy older men (4-6), and no reports

Address reprint requests to Dr. Johannes Veldhuis, Box 202, Division of Endocrinology and Metabolism, Department of Internal Medicine, University of Virginia School of Medicine, Charlottesville, VA 22908.

Received for publication 22 July 1987 and in revised form 21 October 1987.

J. Clin. Invest.

(c) The American Society for Clinical Investigation, Inc.

$0021-9738 / 88 / 04 / 1020 / 10 \$ 2.00$

Volume 81, April 1988, 1020-1029 have delineated the characteristics of episodic bioactive $\mathrm{LH}$ secretion in such individuals. The assessment of bioactive hormone is particularly important, since significant discrepancies can exist between immunoreactive and biologically active hormone concentrations. Moreover, an appraisal of alterations in the pulsatile mode of LH secretion is necessary, since the pulsatile nature of $\mathrm{LH}$ release is a physiological property of normal hypothalamopituitary axis function.

Whereas older men retain reproductive capacity, several studies have delineated a continuous slow decline in serum testosterone concentrations with healthy aging (7-9) and corresponding increases in circulating concentrations of immunoreactive gonadotropins $(7,10,11)$. The latter findings were accompanied by diminished (delayed and blunted) responses of the anterior pituitary gland to a pharmacological injection of exogenous gonadotropin-releasing hormone $(\mathrm{GnRH})^{1}(12)$. Such observations using RIA have suggested possible decreases in pituitary gonadotropin secretory reserve in healthy aging men. Similar information is not available in relation to bioactive $\mathbf{L H}$. In addition, in aging male rats, serum testosterone concentrations fall with age in association with a decrease in serum immunoactive gonadotropin concentrations (13). This diminished release of $\mathrm{LH}$ is associated with a decrease in $\mathrm{LH}$ pulse frequency and amplitude in this species $(14,15)$. Moreover, a recent study in male rats found no statistically significant effects of age on the amount of GnRH released from mediobasal hypothalami perfused under basal conditions and after stimulation with high extracellular potassium. Such data imply that altered function of the hypothalamic-pituitary-gonadal axis in aged male rats is not due to a diminution in GnRH-synthesizing neurons (16).

The development and application of a sensitive and precise in vitro bioassay for human LH (17) has allowed the demonstration that in healthy young men LH is secreted in physiological pulses of high biological activity (1-3). Moreover, by combining bioassay and RIA, the ratio of bioactive to immunoactive LH (B:I ratio) can be used to estimate the quality of LH released under different circumstances (3). Such analyses in men with prostate cancer recently revealed significantly lower plasma B:I LH ratios in older men compared with those found in younger healthy men (4). In subsequent studies of a range of ages of men selected from a healthy population, single blood samples from older individuals were found to have significantly lower B:I LH ratios (5). In addition, acute administration of a maximal dose of the hypothalamic releasing factor, $\mathrm{GnRH}$, to healthy older men did not significantly increase the plasma B:I ratio (18). However, deficient pituitary release of bioactive LH cannot necessarily be inferred from these studies,

1. Abbreviations used in this paper: B:I, bio:immuno; GnRH, gonadotropin-releasing hormone. 


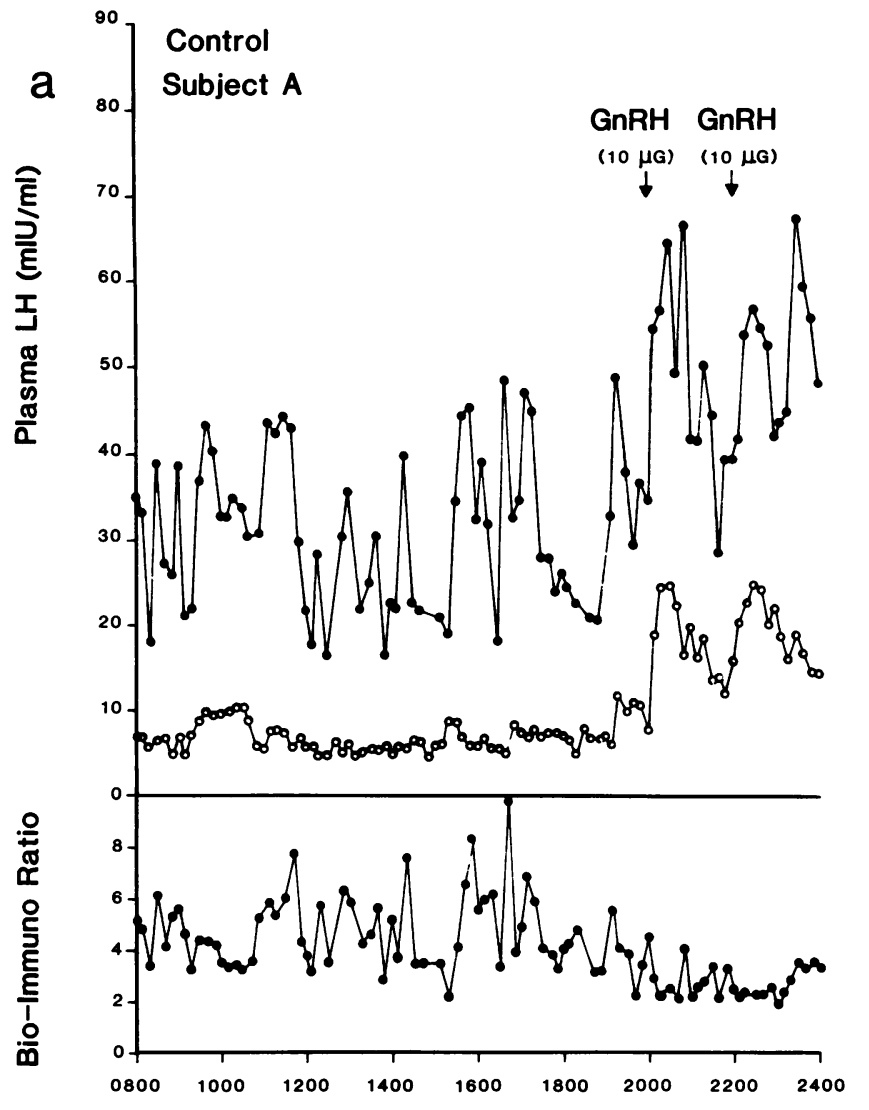

Time (h)

Figure 1. Illustrative profiles of serial plasma bioactive (BIO-) and immunoactive (RIA) LH concentrations and the corresponding B:I LH ratios. Profiles are from two healthy older men, who underwent blood sampling at $10-\mathrm{min}$ intervals for $16 \mathrm{~h} . A$ and $C$ represent the

since we have recently observed that even in younger men, low-dose intravenous pulses of GnRH-but not pharmacological injections-are able to evoke the release of $\mathrm{LH}$ enriched in bioactivity (2).

A recent study in young men using steady state estradiol infusions and oral administration of the antiestrogen, tamoxifen, revealed that endogenous estradiol can significantly modulate the pulsatile secretion of bioactive LH by influencing spontaneous LH pulse frequency, amplitude, and basal (intrapulse and interpulse) B:I LH ratios (3). Since estradiol concentrations typically increase with age, we have considered the following specific hypotheses to account for diminished plasma B:I ratios in healthy men over 60 yr old: $(a)$ the hypothalamic regulation of spontaneous pulsatile secretion of bioactive LH is altered in healthy older men; $(b)$ the direct pituitary release of bioactive $\mathrm{LH}$ in response to intravenous pulses of exogenous GnRH is limited in older men; and/or $(c)$ the above postulated findings are related to negative feedback actions of endogenous estradiol in older men. The latter consideration reflects the ability of exogenous estradiol to preferentially suppress bioactive $\mathbf{L H}$ release in young men with a consequent reduction in the B:I ratio, as well as the recognized decreases in B:I ratios in young estrogenized women compared with their postmenopausal counterparts. To test the preceding

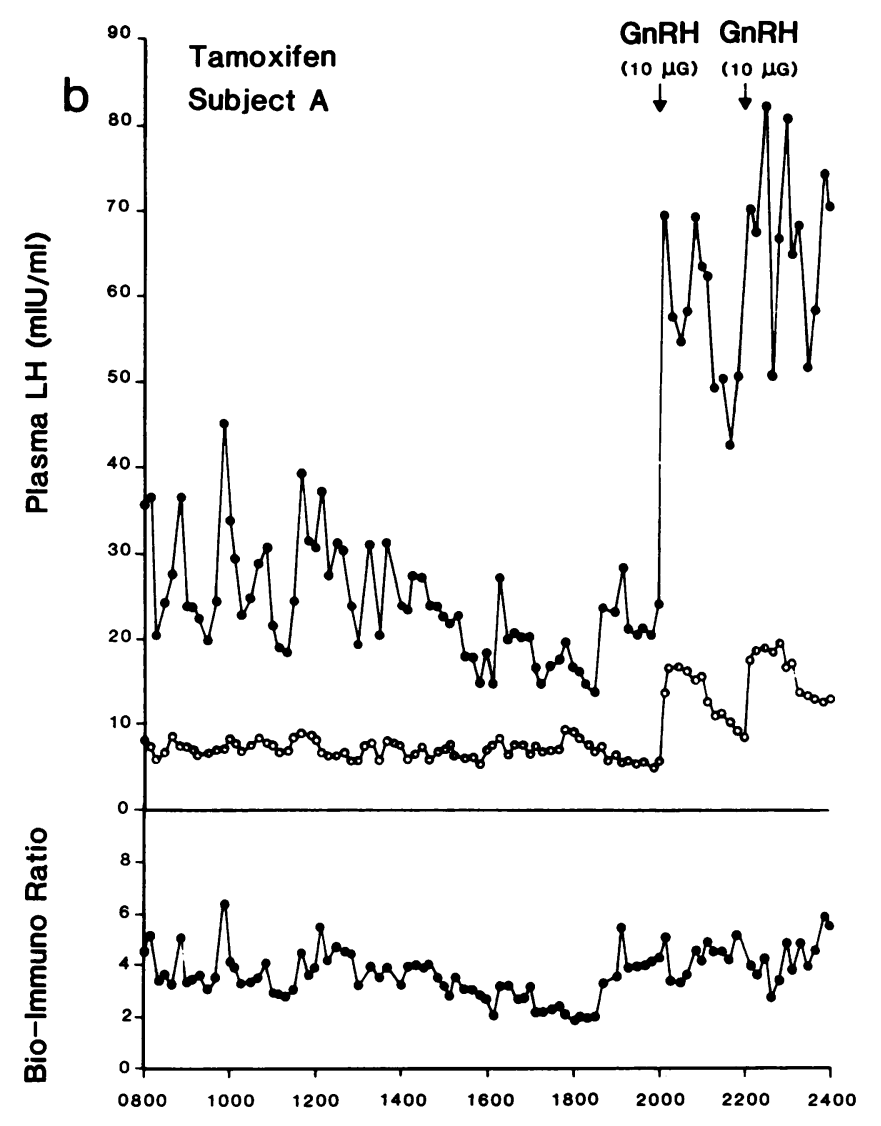

Time (h)

control period, and $B$ and $D$ the periods during tamoxifen treatment. The first $12 \mathrm{~h}$ of basal sampling were followed by two consecutive intravenous pulses of exogenous GnRH $(10 \mu \mathrm{g})$. Bioactive LH concentrations were measured in the rat interstitial-cell testosterone bioassay.

hypotheses, we examined both the quantitative and qualitative properties of spontaneous pulsatile bioactive LH release basally and in response to exogenous GnRH stimulation in six healthy men over $60 \mathrm{yr}$ of age. Physiological characteristics of basal and exogenous GnRH-stimulated bioactive $\mathrm{LH}$ release were investigated in these healthy older men under control conditions and after antiestrogen administration. These analyses permitted us to compare such basal properties and stimulated responses with those identified recently in younger men (3). These studies of bioactive gonadotropin secretory dynamics have unmasked significant alterations in basal and stimulated bioactive LH release in healthy older men compared with their young counterparts.

\section{Methods}

Patient characteristics. Six healthy active men $60-75$ yr of age who had no underlying medical problems, were receiving no medication, and were stable, secure, robust, community-based individuals, participated in this study after providing written informed consent, which was approved by the Human Investigation Committee of the University of Virginia School of Medicine. Each individual had normal serum concentrations of immunoreactive cortisol, thyroxine, prolactin, $\mathrm{LH}$, follicle-stimulating hormone, thyroid-stimulating hormone, free testos- 

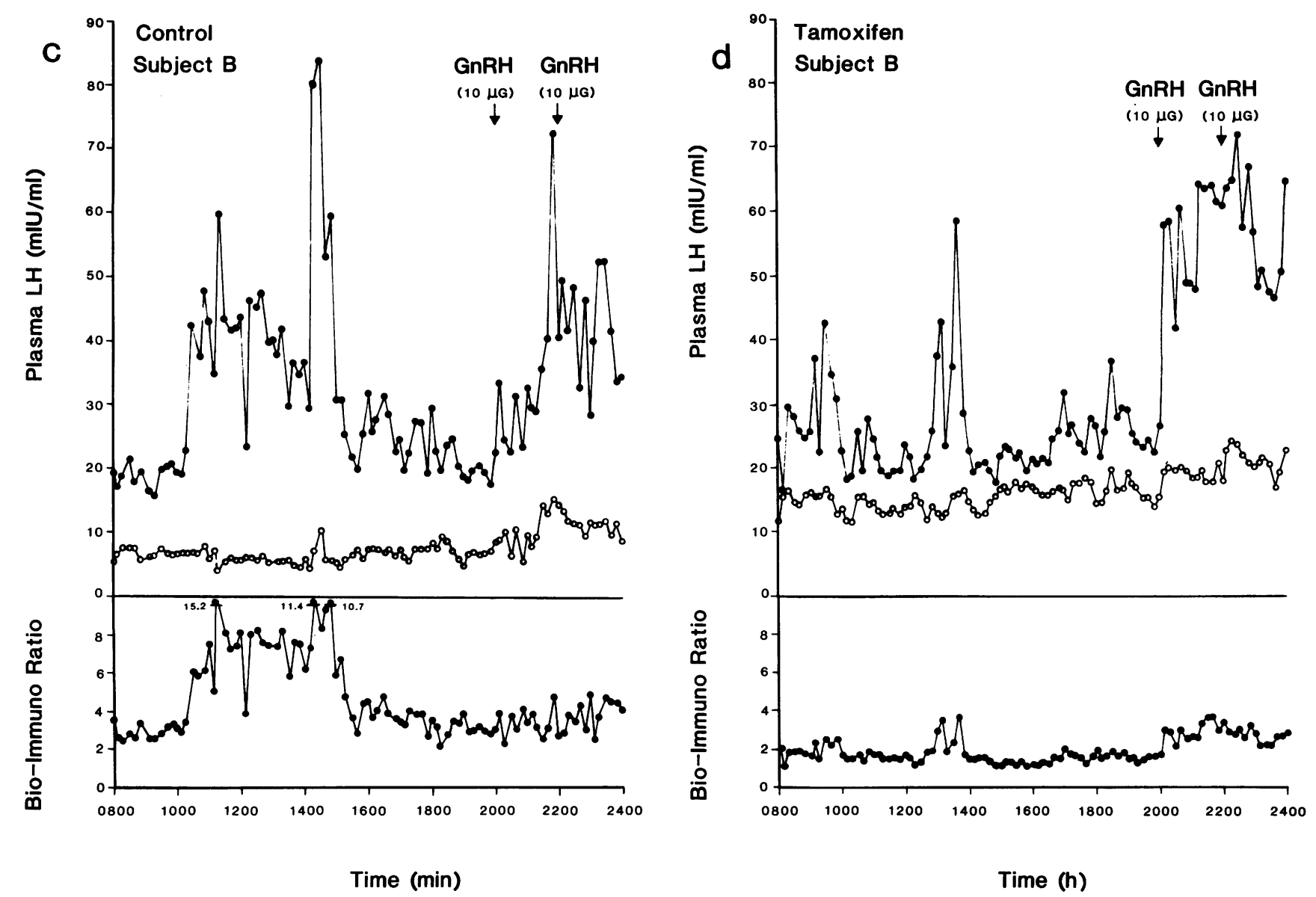

Figure 1 (Continued)

terone, estradiol and normal biochemical tests of hepatic, renal, and hematological functions. Similar characteristics of a control group of young men (age $<30$ ) have been previously described (3).

Clinical protocol. Pulsatile release of LH was studied by drawing blood samples every $10 \mathrm{~min}$ for $16 \mathrm{~h}$ beginning at 0800 hours. After 12 $\mathrm{h}$ of baseline sampling, $10 \mu \mathrm{g} \mathrm{GnRH}$ was injected intravenously. A second $10 \mu \mathrm{g}$-dose of $\mathrm{GnRH}$ was injected $2 \mathrm{~h}$ later. At least 1 mo after this basal (control) sampling procedure, the men were administered 10 mg tamoxifen orally every $12 \mathrm{~h}$ for $7 \mathrm{~d}$. Blood sampling was repeated on the seventh day of antiestrogen administration, in exactly the manner described above. Tamoxifen was continued during the sampling session, with doses given $1 \mathrm{~h}$ before the onset of sampling and $12 \mathrm{~h}$ later.

Hormone assays. Plasma samples were assayed for immunoactive LH by double-antibody RIA with a sensitivity of $1 \mathrm{mIU} / \mathrm{ml}$ in terms of the Second International Reference Preparation of Human Menopausal Gonadotropin (Second IPR), and for bioactive LH by the rat interstitial-cell testosterone bioassay, with a sensitivity of $0.4 \mathrm{mIU} / \mathrm{ml}$. The coefficients of variation were 8.4 (at $2 \mathrm{mIU} / \mathrm{ml}$ ), 6.8 (at 11.5 $\mathrm{mIU} / \mathrm{ml}$ ), and $4.6 \%$ (at $47 \mathrm{mIU} / \mathrm{ml}$ ) for the RIA, which exhibits $<15 \%$ cross-reactivity with alpha subunit $(1,2)$.

Pulse analysis. A computer-based pulse detection method, termed Cluster analysis, was used to quantitate specific properties of pulsatile LH release objectively (19). This algorithm identifies all significant increases (peak upstrokes) and decreases (peak downstrokes) in the data in relation to dose-adjusted variance. Accordingly, pulse frequency and amplitude, peak duration and area, interpeak intervals (minutes), and interpeak concentrations (milli-International Units per milliliter) are calculated. The following program parameters were used to constrain the analysis to a $1 \%$ false-positive rate on signal-free noise: (a) test cluster sizes of two points for nadir and two points for peak; $(b)$ two degrees of freedom per sample mean; $(c)$ use of an $8 \%$ median within-assay coefficient of Variation for the rat interstitial-cell testosterone, and $(d) t$ statistics of 2.75 for a significant increase and 1.5 for a significant decrease. The same parameters were used for the younger men previously reported (3).

Statistical analysis. Deconvolution analysis was carried out as previously described, in order to estimate bioactive $\mathrm{LH}$ secretion rates in response to exogenous GnRH pulses (20). For normally distributed data (means and 12-h integrated values), within-subject differences were sought by paired two-tailed $t$ testing and between subject differences assessed by unpaired two-tailed (equal variance) $t$ testing. Because of departures from normality, individual pulse parameters were compared using the Wilcoxon ranked sign (paired) or Wilcoxon rank sum (unpaired) tests. Results are expressed as means \pm SEM or median values. Statistical significance was construed for $P \leq 0.05$.

\section{Results}

\section{Characteristics of spontaneous (basal) and GnRH-induced} bioactive $L H$ release in older men

Healthy older men had a mean basal bioactive $\mathrm{LH}$ pulse frequency of $6.0 \pm 0.3$ pulses $/ 12 \mathrm{~h}$ with a mean interpulse interval of $92 \pm 6.0 \mathrm{~min}$. The mean peak duration was $78 \pm 5.2 \mathrm{~min}$ with a maximal peak height of $44 \pm 3 \mathrm{mIU} / \mathrm{ml}$. The $12-\mathrm{h}$ integrated and mean $\mathrm{LH}$ concentrations were $21,500 \pm 1,400 \mathrm{mIU} / \mathrm{ml} /$ 
Table I. Characteristics of Spontaneous Bioactive LH Pulsatility in Healthy Older Men before and after Tamoxifen Treatment

\begin{tabular}{|c|c|c|}
\hline $\begin{array}{c}\text { LH pulse } \\
\text { Characteristic }\end{array}$ & Control & Tamoxifen \\
\hline Frequency (pulses $/ 12 \mathrm{~h}$ ) & $6.0 \pm 0.3(6)^{*}$ & $7.8 \pm 0.5(8)^{\ddagger}$ \\
\hline Interpulse interval (min) & $92 \pm 6.0(86)$ & $84 \pm 5.4(81)$ \\
\hline Peak duration (min) & $78 \pm 5.2(82)$ & $58 \pm 5 \quad(54)^{\ddagger}$ \\
\hline $\begin{array}{l}\text { Maximum peak height } \\
\qquad(\mathrm{mI} U / \mathrm{ml})\end{array}$ & $44 \pm 3$ & $39 \pm 4 \quad(37)$ \\
\hline Peak area $(\mathrm{mIU} / \mathrm{ml} / \mathrm{min})$ & $870 \pm 114(762)$ & $500 \pm 84(430)^{\ddagger}$ \\
\hline $\begin{array}{l}\text { Incremental peak } \\
\text { amplitude }(\mathrm{mIU} / \mathrm{ml})\end{array}$ & $20 \pm 2.3$ & $14 \pm 1.6(12.1)^{\ddagger}$ \\
\hline $\begin{array}{l}\text { Interpeak valley } \\
\text { duration ( } \mathrm{min} \text { ) }\end{array}$ & $23 \pm 1.6(22.2)$ & $25 \pm 2.0(24.0)$ \\
\hline $\begin{array}{l}\text { Interpeak valley mean } \\
(\mathrm{mIU} / \mathrm{ml})\end{array}$ & $26 \pm 1.3(26.2)$ & $27 \pm 2.2(26)$ \\
\hline
\end{tabular}

* Data are mean \pm SEM (median) values for six men sampled at 10 min intervals for $12 \mathrm{~h}$ basally (control) and after administration of the antiestrogen, tamoxifen. Pulsatility was evaluated by Cluster analysis.

$\ddagger P<0.05$ vs. control by paired nonparametric tests.

min and $31 \pm 2.0 \mathrm{mIU} / \mathrm{ml}$, respectively. Plasma B:I LH ratios had a grand median value of $2.7 \pm 0.4$, a median interpulse ratio of $2.4 \pm 0.03$, and a median intrapulse ratio of $2.9 \pm 0.5$. In response to exogenous $\mathrm{GnRH}$ pulses (administered at $10 \mu \mathrm{g}$ doses each $2 \mathrm{~h}$ apart), older men increased their hourly mean bioactive $\mathrm{LH}$ concentrations $(\mathrm{mIU} / \mathrm{ml})$ from preGnRH baselines of $25.2 \pm 4.2$ (h 1 ) and $27.7 \pm 4.4$ (h 2 ) to GnRH-stimulated values of $67.5 \pm 13.1$ (h 3), $64.6 \pm 12.1$ (h 4), $65.4 \pm 10.0$ (h 5), and $64.7 \pm 10.6(\mathrm{~h} 6)$. The corresponding B:I ratios were baseline $2.3 \pm 0.5$ and $2.3 \pm 0.4$, and GnRH-stimulated $2.5 \pm 0.3$, $2.6 \pm 0.3,2.4 \pm 0.4$, and $2.7 \pm 0.4(P=N S)$. Two profiles of bioactive $\mathrm{LH}$ release (basal and exogenous GnRH-stimulated) are illustrated in Fig. 1, and discussed further below.
Alterations in integrated, pulsatile, and exogenous GnRHstimulated bioactive $L H$ release in response to the antiestrogen, tamoxifen

Integrated bioactive $L H$ concentrations. The $12-\mathrm{h}$ mean and integrated plasma bioactive $\mathrm{LH}$ concentrations in older men were $31 \pm 2.0 \mathrm{mIU} / \mathrm{ml}$ and $21,500 \pm 1,400 \mathrm{mIU} / \mathrm{ml}$ per min at baseline, and $30 \pm 2.8 \mathrm{mIU} / \mathrm{ml}$ and $21,450 \pm 1,900 \mathrm{mIU} / \mathrm{ml}$ per min after treatment with tamoxifen. There were no statistically significant changes in bioactive $\mathrm{LH}$ concentrations in response to tamoxifen $(P=0.96$ [concentration], $P=0.91$ [mean]).

Characteristics of pulsatile bioactive $L H$ release. Table I summarizes the characteristics of pulsatile bioactive $\mathrm{LH}$ release observed in six older healthy men at baseline and after treatment with tamoxifen. Comparison of pulse characteristics in the baseline and tamoxifen-treated sessions revealed the following significant effects of tamoxifen: $(a)$ Increased bioactive LH pulse frequency, and $(b)$ decreased bioactive LH peak duration, incremental peak amplitude, and peak area. Fig. 2 shows the individual changes in these pulse characteristics in the six men in response to antiestrogen administration.

Plasma B:I LH ratios. Table II summarizes the plasma B:I LH ratios in four different experimental contexts: (a) the overall basal (12-h) median B:I ratio; (b) the median B:I ratio calculated from all $\mathrm{LH}$ values not part of a spontaneous peak, i.e., interpeak values; (c) the median B:I ratio calculated from all points contained within a spontaneous peak, i.e., intrapeak values; and (d) the median B:I ratio within the LH peaks induced by pulsed exogenous GnRH administration. Both spontaneous bioactive LH peaks and exogenous GnRH-induced LH peaks had significantly higher B:I ratios than the interpeak basal values. Comparison of the control and tamoxifen groups showed no significant effects of tamoxifen on any of the four subgroups of B:I ratios.

Bioactive $L H$ release in response to consecutive pulses of exogenous $\mathrm{GnRH}$. To assess responses to exogenous GnRH, we analyzed the $2 \mathrm{~h}$ of baseline sampling immediately preceding the two consecutive IV GnRH boluses, and the subsequent 4-h period containing the $\mathrm{LH}$ responses to exogenous $\mathrm{GnRH}$. Responses to exogenous pulses of $\mathrm{GnRH}$ were compared in
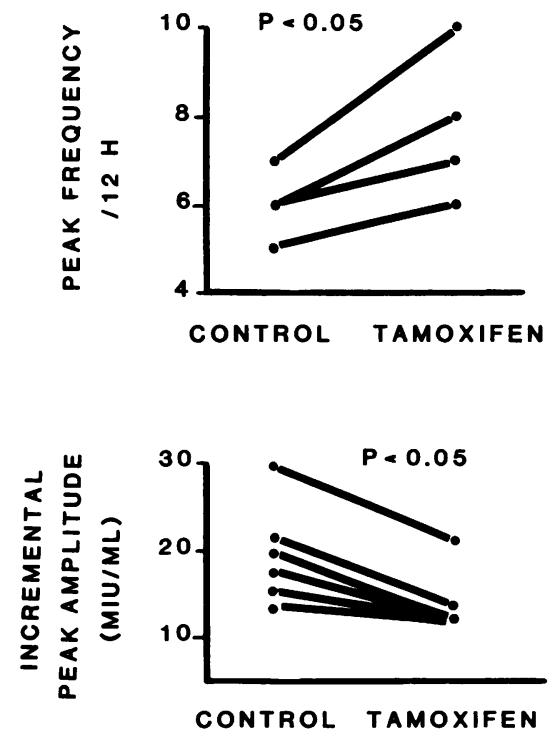
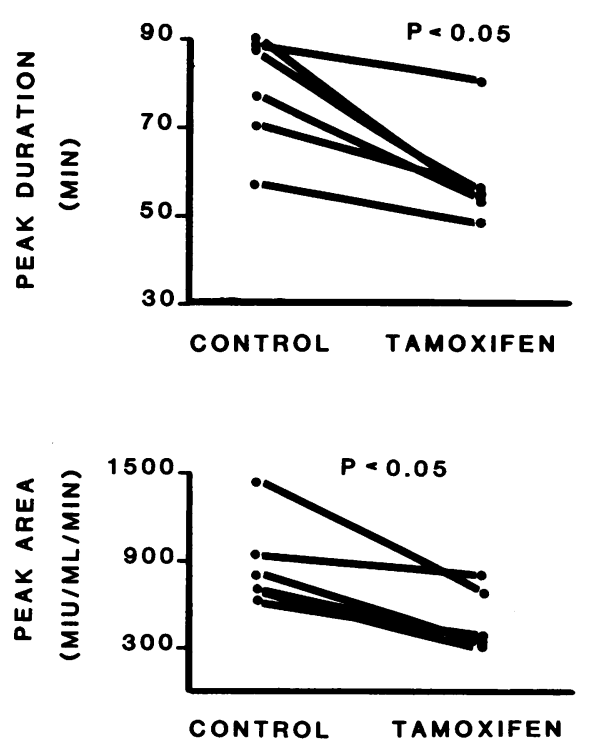

Figure 2. Statistically significant changes in pulse characteristics in six healthy older men ( $>60 \mathrm{yr}$ old) basally and after the administration of an antiestrogen, tamoxifen. 
Table II. Summary of Median Plasma B:I LH Ratios in Healthy Older Men (> $60 \mathrm{Yr}$ Old) before and after Tamoxifen

\begin{tabular}{llll}
\hline \multicolumn{1}{c}{ Plasma B/I ratios } & Control & Tamoxifen & $P$ value* \\
\hline $\begin{array}{l}\text { Overall basal } \\
\text { (12-h) }\end{array}$ & $2.7 \pm 0.4$ & $2.2 \pm 0.3$ & 0.25 \\
Interpulse value & $2.4 \pm 0.3$ & $2.1 \pm 0.3$ & 0.52 \\
Intrapulse value & $2.9 \pm 0.5^{\ddagger}$ & $2.3 \pm 0.3^{\ddagger}$ & 0.17 \\
GnRH-stimulated & $2.5 \pm 0.3$ & $2.9 \pm 0.3^{\ddagger}$ & 0.41
\end{tabular}

* $P$ value for comparison between control and tamoxifen groups. Data are means \pm SEM $(n=6$ men).

${ }^{\ddagger} P<0.05$ vs. corresponding interpulse values.

the control and tamoxifen treatment sessions using hourly means for plasma bioactive LH concentrations and hourly medians for B:I ratios. Fig. 3 depicts the temporal profile of bioactive and immunoactive $\mathrm{LH}$ release and the associated B:I ratios in older men in response to exogenous paired $\mathrm{GnRH}$ pulses. In the group of older men, whether studied in the absence or presence of tamoxifen, exogenous $\mathrm{GnRH}$ injections significantly increased bioactive and immunoactive LH concentrations and the mean plasma B:I LH ratio. However, three individual older men responded sparingly (or not at all) to GnRH, as illustrated in Fig. 1. Tamoxifen did not significantly modify these responses to exogenous GnRH for the group of six older men as a whole, but the three older men with absent basal responses to GnRH exhibited significantly facilitated responses during tamoxifen treatment. This pattern of tamoxifen facilitated exogenous GnRH action as illustrated in Fig. 1.

Comparison of spontaneous (baseline) pulsatile bioactive $L H$ release in older men and younger men

12-h integrated and mean plasma bioactive LH concentrations. The 12-h mean and integrated bioactive LH concentrations were not significantly different between young and older men $(P=0.23$ and 0.26 , respectively).

Characteristics of pulsatile $\mathrm{LH}$ release. Bioactive $\mathrm{LH}$ release in young men (ages 21-30) was evaluated in a manner identical to that in the present group of older men (ages 60-75). Although several individual older men exhibited irregular bursts of bioactive $\mathrm{LH}$ release not observed in young men (Fig. 1), detailed statistical comparisons of individual pulse properties of spontaneous bioactive LH release in the young and older groups revealed no statistically significant differences in mean spontaneous bioactive LH pulse frequency, interpulse interval, peak duration, maximal peak height, incremental peak amplitude, or bioactive $\mathrm{LH}$ peak area. The estimated type II (beta) error for a $30 \%$ difference in this comparison at an alpha level of 0.05 was $<0.1$; viz., the power of the test was $>90 \%$ for detecting a significant difference between young and older men, if such a difference were present.

Plasma B/I LH ratios. Comparison of median plasma B:I ratios revealed the following similarities and differences between young and older men: $(a)$ overall median B:I ratios were similar $(P=0.89) ;(b)$ interpulse B:I ratios were similar $(P$ $=0.51) ;(c)$ intrapulse B:I ratios were similar $(P=0.99)$; but $(d)$ stimulated B:I ratios were different $(P=0.05)$, in that younger men had higher B:I ratios than older men.

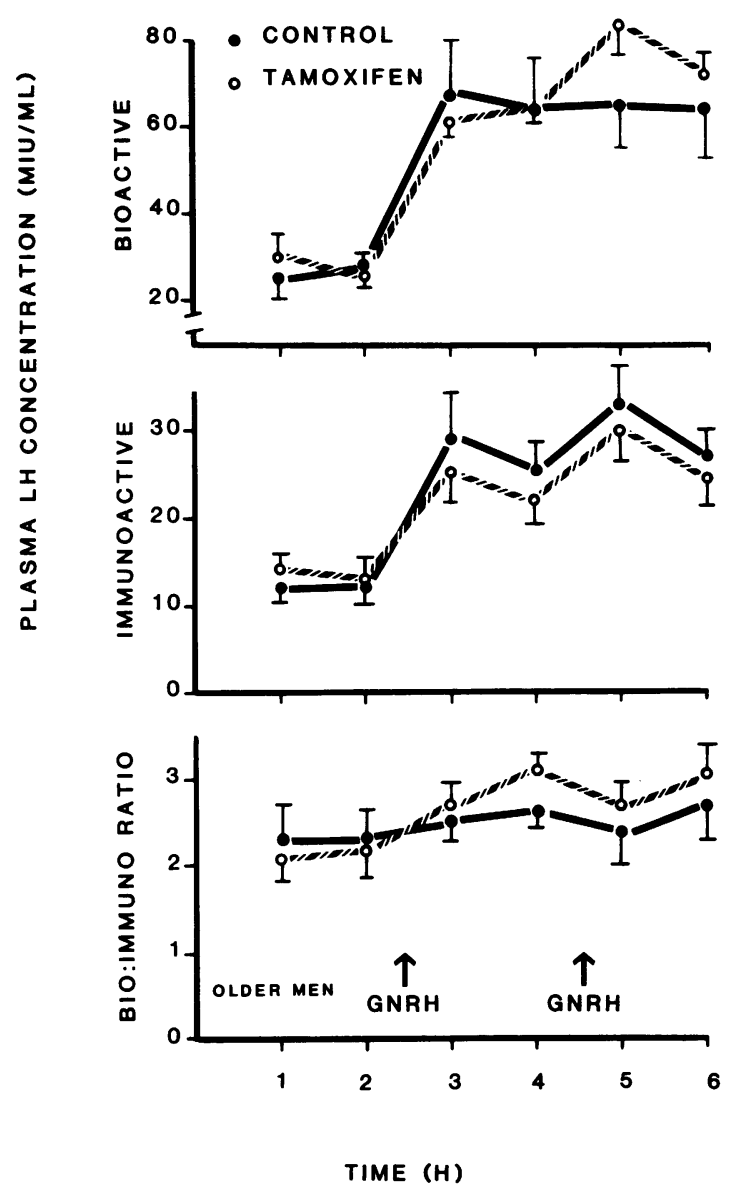

Figure 3. Temporal profiles of hourly mean plasma bioactive $\mathrm{LH}$ concentrations (top), immunoactive LH concentrations (middle), and associated B:I LH ratios in older men in response to two consecutive intravenous pulses of exogenous GnRH $(10 \mu \mathrm{g})$. Data are means $\pm \operatorname{SEM}(n=6$ men $)$.

Bioactive $L H$ release in response to exogenous $G n R H$. Statistically significant differences were observed between young and older men in relation to mean hourly bioactive LH concentrations following GnRH administration. Although basal ( 1 and $2 \mathrm{~h}$ prior to $\mathrm{GnRH}$ ) bioactive $\mathrm{LH}$ concentrations were similar in the two groups $(P>0.132)$, during $\mathrm{GnRH}$ administration (hours 3-6), the following significant differences (and the corresponding $P$ values) emerged between younger and older men, with older men having significantly lower values than younger men: at $3 \mathrm{~h}, P=0.02$; at $4 \mathrm{~h}, P=0.01$; at $5 \mathrm{~h}, P$ $=0.002$; and at $6 \mathrm{~h}, P=0.025$.

Similarly, plasma B:I LH ratios in older and young men were not significantly different during the $2 \mathrm{~h}$ preceding GnRH injections $(P \geq 0.54)$, but manifested significant differences in all but the last hour during $\mathrm{GnRH}$ administration $(P$ $=0.04,0.01,0.03,0.08$ for $\mathrm{h} 2-5$, respectively). Fig. 4 presents the time course of these differences in plasma bioactive LH concentrations and plasma B:I LH ratios in the two groups.

\section{Differences between older and young men in response to tamoxifen}

12-h integrated and mean bioactive $L H$ concentrations in older and young men. Older men exhibited $~ 50 \%$ lower 12-h integrated bioactive LH concentrations in the presence of tamoxi- 

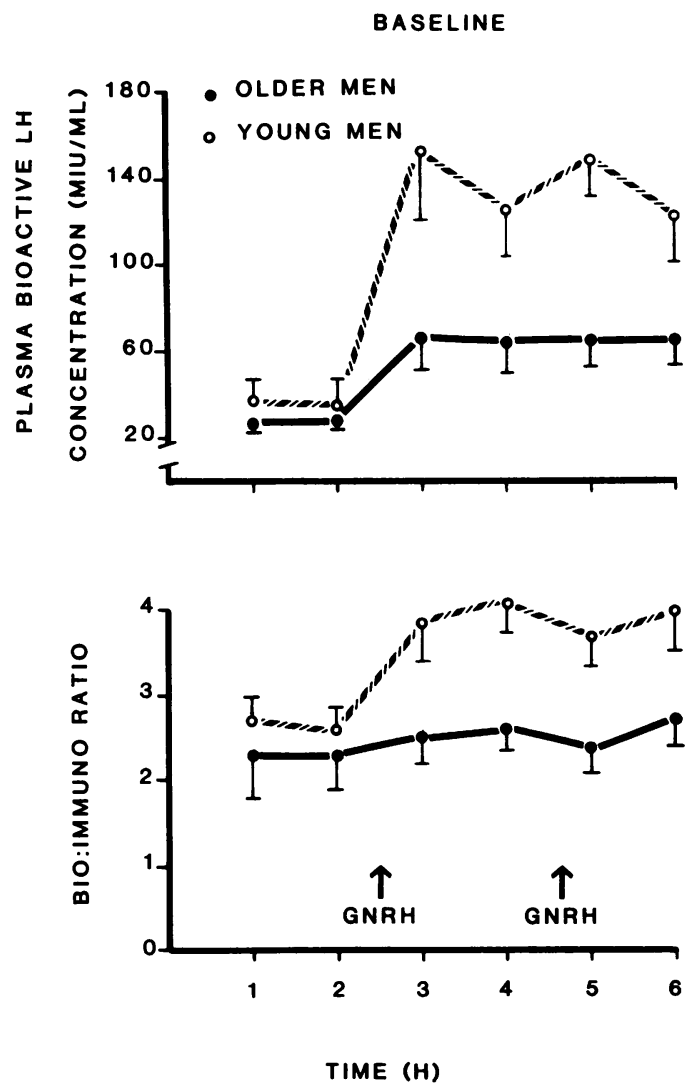

Figure 4. Temporal profiles of hourly mean plasma bioactive $\mathrm{LH}$ concentrations (top) and B:I LH ratios in young and older men on the control day, basally and in response to paired pulses of exogenous $\mathrm{GnRH}$. Data are means $\pm \operatorname{SEM}(n=6 \mathrm{men})$.

fen than younger men $(42,044 \pm 8,200$ vs. $21,450 \pm 1,900 \mathrm{mIU} /$ $\mathrm{ml}$ per min, $P=0.03)[P<0.01]$. Older men also had similarly lower 12-h mean bioactive $\mathrm{LH}$ concentrations on tamoxifen than younger men $(58.6 \pm 11.3 \mathrm{mIU} / \mathrm{ml}$ vs. $30.4 \pm 2.8 \mathrm{mIU} / \mathrm{ml}$, $P=0.04$ ).

Characteristics of tamoxifen-stimulated pulsatile bioactive $L H$ release in older and young men. The individual properties of pulsatile bioactive $\mathrm{LH}$ release in young and older men treated with tamoxifen were statistically similar for the following parameters: pulse frequency, interpulse interval, peak duration, peak height, or peak area. However, older men had $\sim 50 \%$ lower incremental bioactive LH pulse amplitudes compared with younger men; i.e., older men had a mean incremental bioactive LH peak amplitude of only $14.0 \pm 1.6$ $\mathrm{mIU} / \mathrm{ml}$, whereas younger men had a value of $27.0 \pm 4.1 \mathrm{mIU} /$ $\mathrm{ml}(P<0.05)$.

Comparison of the influence of tamoxifen on plasma bio:immuno $\mathrm{LH}$ ratios in older and young men. Older men on tamoxifen exhibited significantly lower plasma B:I LH ratios than young men in the following respects: $(a)$ overall median basal B:I ratios $(P=0.02) ;(b)$ interpulse B:I ratios $(P=0.01)$; and $(c)$ intrapulse B:I ratios $(P=0.03)$, but not GnRH-stimulated $\mathrm{B}: \mathrm{I}$ ratios $(P=0.25)$.

Comparison of the effects of tamoxifen on GnRH-stimulated bioactive $L H$ release in older and young men. Although older men exhibited significantly impaired responses to GnRH in the basal state (no tamoxifen) compared with young men, treatment with tamoxifen altered some of these differences. As shown in Fig. 5, during treatment with tamoxifen the release of bioactive LH in response to the first (compared with the second) pulse of exogenous GnRH was not diminished in older men as it was in younger men receiving tamoxifen. However, older men failed to show the same degree of additional increase in bioactive $\mathrm{LH}$ concentrations observed in young men in response to the second exogenous GnRH bolus $(P=0.04)$. Moreover, there were no statistically significant differences between B:I ratios in young and older men over the $4 \mathrm{~h}$ of $\mathrm{GnRH}$ administration while on tamoxifen $(P=0.21,0.36,0.10$, and 0.28 .

\section{Deconvolution of the GnRH-stimulated bioactive LH pulses}

To estimate possible differences in endogenous bioactive $\mathrm{LH}$ secretory rates in older and young men given exogenous GnRH pulses, we used deconvolution analysis (20). The latter method permits one to estimate underlying secretion rates from serial plasma hormone concentrations. Figs. $6 A$ and $B$ depict bioactive LH concentration peaks (upper) and the calculated underlying secretory episode after IV GnRH pulses in the six younger men $(A)$ and six older men $(B)$ as analyzed by deconvolution-both under basal conditions and after treatment with tamoxifen. The quantitative results are summarized in Table III. In general, older men exhibited significantly lower calculated bioactive LH secretory rates (and mass) than younger men in response to GnRH administration. In addition, older men did not manifest the response pattern observed in young men; i.e., young but not older men showed a relatively diminished secretory response to the first compared to

\section{TAMOXIFEN}
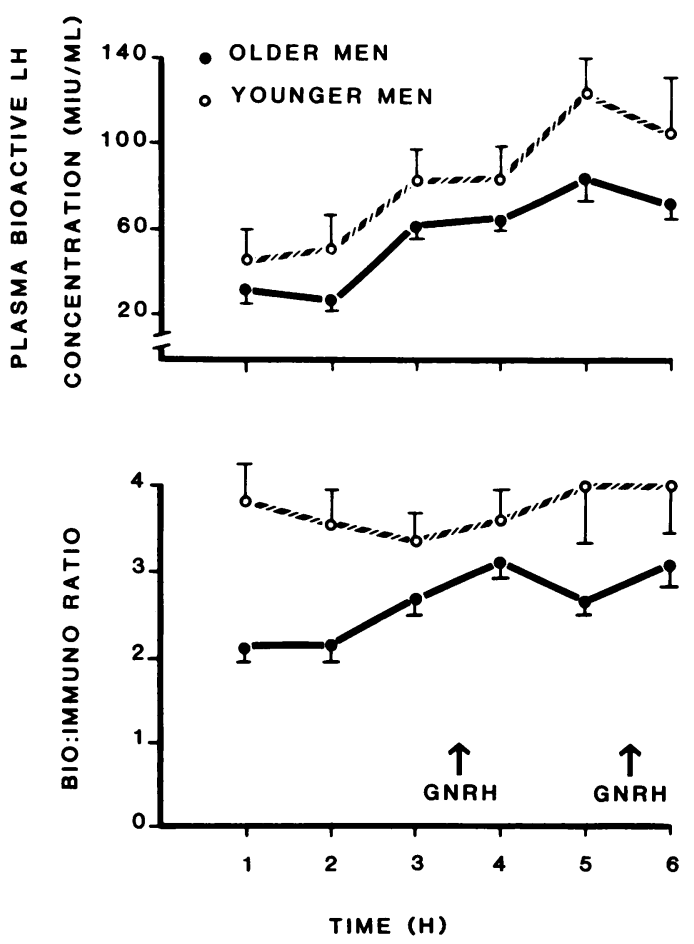

Figure 5. Temporal profiles of hourly mean plasma bioactive $\mathrm{LH}$ concentrations (top) and B:I LH ratios (bottom) in younger and older men during treatment with an antiestrogen, tamoxifen. Results are otherwise as given in the legend of Fig. 3. 

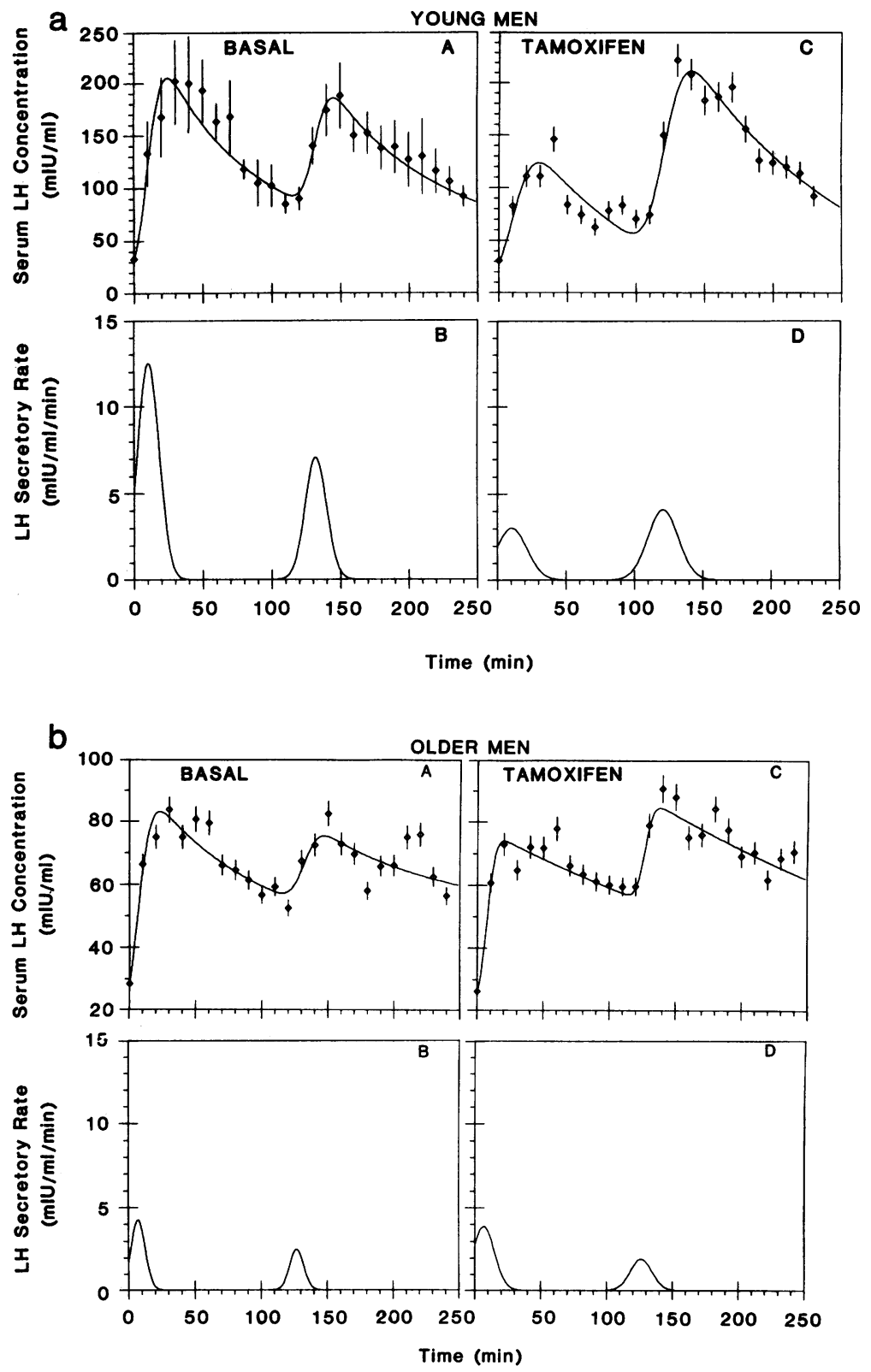

Figure 6. Bioactive LH concentration peaks and calculated underlying secretory episodes in response to two consecutive intravenous pulses of $10 \mu \mathrm{g}$ exogenous GnRH in six young men $(a)$ and six older men $(b)$. Plasma bioactive LH concentrations $(A$ and $C)$ are given as means $\pm \operatorname{SEM}(n=6$ men), for which the accompanying mean calculated reconvolution curves are shown. Secretory episodes are plotted as mean regressions of secretory rates vs. time ( $B$ and $D$ ) which were estimated by multiple-parameter deconvolution. Data in young and older men are presented in both the basal (control) and tamoxifen-treated state. Time zero denotes the time when the first of two consecutive intravenous injections of $10 \mu \mathrm{g}$ exogenous $\mathrm{GnRH}$ were administered. Note reduced ordinate scale for older men.

the second bolus of GnRH while on tamoxifen. The exact basis for this pattern is not known, but might reflect partial estrogen agonist effects observed in young but not older men given tamoxifen.

\section{Comparison of sex steroid-hormone concentrations in young and older men}

Differences between circulating sex-steroid hormone concentrations (estradiol and total testosterone) were also sought in young and older men before and after tamoxifen treatment. Statistical analysis revealed no significant differences in sex steroid concentrations between control and tamoxifen groups in older men, or between the younger and older men either basally or after tamoxifen treatment (Table IV). It should be noted that young men did increase their total testosterone, while older men maintained approximately the same total testosterone level. This disparity was significant, $P=0.03$ (Wilcoxon rank sum test).

\section{Discussion}

The present investigations of the dynamics of bioactive $\mathrm{LH}$ release in healthy older men (ages 60-75) have revealed signifcant attenuation of the pituitary's capacity to release biologically active gonadotropic hormone. This diminution of bioactive $\mathrm{LH}$ release could be unmasked by maneuvers designed to enhance endogenous secretion of LH enriched in biological activity. Such evocative procedures consisted of mimicking endogenous $\mathrm{GnRH}$ action by consecutive intravenous pulses of low-dose (10- $\mu \mathrm{g})$ exogenous GnRH, or by presumptively augmenting endogenous GnRH secretion with antiestrogen treatment. In contrast, basal bioactive LH release (studied in the absence of stimulatory procedures) was not significantly impaired in this group of healthy older men, whether assessed as integrated plasma concentrations or quantitative estimates of pulsatile LH release. Since the probability of missing a significant difference in the latter assessments can be estimated as 
Table III. Deconvolution Estimates of Bioactive LH Secretion in Response to Paired Intravenous Pulses of Exogenous GnRH in Young and Older Men

\begin{tabular}{|c|c|c|}
\hline \multicolumn{3}{|c|}{ Maximal bioactive LH secretory rate after GnRH injection } \\
\hline & Young & Older \\
\hline & \multicolumn{2}{|c|}{$\mathrm{mIU} / \mathrm{ml} \cdot \min$} \\
\hline \multicolumn{3}{|l|}{ Basal } \\
\hline GnRH-1* & $13(10-16)^{\ddagger}$ & $4.2(3.4-5.1)^{8}$ \\
\hline GnRH-2* & $7.1(5.5-8.7)$ & $1.3(0.96-1.7)^{\S}$ \\
\hline \multicolumn{3}{|l|}{ Tamoxifen } \\
\hline GnRH-1 & $3.0(2.8-3.2)$ & $4.3(4.2-4.6)^{8}$ \\
\hline GnRH-2 & $4.1(3.7-4.4)$ & $2.5(2.1-3.0)^{8}$ \\
\hline \multicolumn{3}{|c|}{ Calculated mass of bioactive $\mathrm{LH}$ secreted after $\mathrm{GnRH}$ injection } \\
\hline & \multicolumn{2}{|c|}{$m I U / m l$} \\
\hline \multicolumn{3}{|l|}{ Basal } \\
\hline GnRH-1 & $247(196-311)$ & $87(70-107)^{8}$ \\
\hline GnRH-2 & $139(108-171)$ & $27(20-35)^{8}$ \\
\hline \multicolumn{3}{|l|}{ Tamoxifen } \\
\hline GnRH-1 & $85(79-91)$ & $57(55-61)^{8}$ \\
\hline GnRH-2 & $114(104-123)$ & $33(28-39)^{8}$ \\
\hline
\end{tabular}

* GnRH-1 and GnRH-2 refer to consecutive intravenous pulses of exogenous $\mathrm{GnRH}(10 \mu \mathrm{g})$ at 2-h intervals.

${ }^{\ddagger}$ Data are mean parameter estimates with $67 \%$ confidence limits (see Methods and [20]).

${ }^{\S} P<0.05$ for comparison between young vs. older men.

$<10 \%$ (type II error), our observations indicate that in older men the reserve capacity of the hypothalamopituitary axis to secrete increased amounts of biologically active $\mathrm{LH}$ is reduced, but the degree of reduction in bioactive LH reserve is not sufficient to become manifest in the basal (unstimulated) state.

We used two complementary strategies to examine the dynamic responses of the hypothalamopituitary gonadal axis in healthy older men. First, consecutive intravenous pulses of low-dose $(10-\mu \mathrm{g})$ exogenous GnRH were employed to assess the release of LH enriched in biological activity. Unlike continuous infusions or bolus pharmacological doses of exogenous GnRH $(21,22)$, consecutive intravenously pulsed submaximal doses of $\mathrm{GnRH}$ elicit preferential release of bioactive LH with a resultant significant increase in the plasma B:I LH ratio (2). The latter changes presumably reflect the accelerated

Table IV. Serum Steroid-Hormone Concentrations in Young and Older Men

\begin{tabular}{lccc}
\hline \multicolumn{1}{c}{ Steroid hormone } & Young & Older & $P$-value \\
\hline Total testosterone $(\mathrm{ng} / \mathrm{dl})$; basal & $488 \pm 49^{\ddagger}$ & $488 \pm 47$ & 0.99 \\
Total testosterone $(\mathrm{ng} / \mathrm{dl})$; tamoxifen & $692 \pm 113$ & $471 \pm 43$ & 0.09 \\
Estradiol $(\mathrm{pg} / \mathrm{ml})$; basal & $21 \pm 1.9$ & $37 \pm 11$ & 0.19 \\
Estradiol $(\mathrm{pg} / \mathrm{ml})$; tamoxifen & $26 \pm 5.2$ & $27 \pm 5.4$ & 0.93
\end{tabular}

* Comparisons of total testosterone (basal vs. tamoxifen) or estradiol (basal vs. tamoxifen) yielded $P=0.68$ and 0.29 , respectively.

$\ddagger$ Values are means \pm SEM ( $n=6$ men in each group). release by gonadotropes of $\mathrm{LH}$ molecules enriched in specific carbohydrate residues with consequently enhanced bioactivity. A similar pattern of augmented LH bioactivity has been recognized in spontaneous (endogenous GnRH-driven) $\mathrm{LH}$ pulses (3). Based upon these earlier observations, we used this paradigm of paired intravenous pulses of exogenous GnRH to probe for alterations in bioactive $\mathrm{LH}$ release in older men. Our results indicate that although older men can increase their B:I LH ratios to a significant degree in response to exogenously pulsed $\mathrm{GnRH}$, the degree of increase at various times after GnRH is significantly reduced $(0.002<P<0.02)$ compared with that in younger men.

A second strategy for evaluating dynamic responses of the hypothalamopituitary axis consisted of enhancing endogenous GnRH stimulation of gonadotropes. This can be accomplished presumptively by reversible blockade of endogenous inhibitory pathways, e.g. the endogenous opiate or sex-steroid feedback systems (23). Accordingly, administration of naltrexone, an opiate-receptor antagonist, or tamoxifen, an estrogenreceptor antagonist, results in increased pulsatile release of bioactive LH in man, presumably reflecting augmentation of endogenous $\mathrm{GnRH}$ release $(3,23)$. When healthy older men were given tamoxifen, they responded by increasing bioactive LH pulse frequency to the same degree as young men, indicating adequate absorption of tamoxifen as well as intact responses of the hypothalamopituitary axis to blockade of the endogenous estrogen-mediated, negative feedback system. However, older men were unable to augment bioactive $\mathrm{LH}$ incremental pulse amplitude to the same degree as young men. If LH pulse amplitude is an index of GnRH action as well as gonadotrope responsiveness, we can infer that the failure of older men to maximally enhance endogenous bioactive $\mathrm{LH}$ release reflects an attenuation in either the amount of GnRH released per pulse by the hypothalamus (despite an increase in the frequency of GnRH pulses), and/or a decrease in gonadotrope responsiveness. The latter consideration is strongly supported by our observation that basal (control) gonadotrope responsiveness to exogenous GnRH pulses in older men is significantly reduced. These age-related alterations in gonadotropin release were not associated with prominent differences in the sex-steroid hormone milieu in young and older men, inasmuch as plasma estradiol and testosterone concentrations were similar in the two groups basally. Nonetheless, after tamoxifen administration, older men failed to increase total testosterone concentrations to the same degree as young men.

The present results in healthy aging men are comparable in certain, but not all, respects with observations in experimental animals. For example, aging male rats show diminished serum testosterone and LH concentrations compared with younger rodents, whereas healthy older men do not show consistent or marked androgen deficiency and tend to have normal or slightly increased serum immunoactive gonadotropin concentrations $(12,13)$. In older rats, spontaneous $\mathrm{LH}$ pulses are of diminished amplitude (14), while in the human, basal LH pulse characteristics are similar in younger and older age groups (present data). However, when the hypothalamopituitary axis of the aged male rat is further stimulated by either withdrawal of negative feedback (castration) or exogenous GnRH administration, a diminished capacity for $\mathrm{LH}$ release can be unmasked $(13,15)$. The present study in healthy aging men has also demonstrated diminished LH responsiveness to neuroendocrine stimuli achieved by opposition of negative 
feedback (antiestrogen administration) or exogenous GnRH injections. Since similar concentrations of hypothalamic GnRH have been documented in old and young rat brains (16), attenuated gonadotrope secretory reserve may represent an age-related change in the gonadal axis common to both man and rat. We would emphasize, however, that to date, determinations of $\mathrm{LH}$ release in the aging rat have been restricted to assessments of immunoactive LH pulsatility, while the current study has allowed an evaluation of bioactive LH release.

The preceding results indicate that the reserve secretory capacity of pituitary gonadotropes for bioactive LH release is significantly attenuated in healthy aging men, whether this reserve capacity is tested by stimulation with exogenous or endogenous GnRH. However, this inference depends upon the assumption that the metabolic clearance rate of bioactive $\mathrm{LH}$ is similar in young and older men. Although the rate of metabolic removal of bioactive LH has been estimated recently in younger men (24), possible age-dependent alterations in the metabolic clearance of bioactive LH in man have not been described. Accordingly, as an indirect test of this consideration, we applied a multiple-parameter deconvolution technique, in which endogenous secretion and clearance rates can be estimated simultaneously for one or more LH peaks (20). This deconvolution procedure permits one to determine the amplitude and half-duration of the underlying secretory impulse, after mathematical removal of the resolved clearance rate. Such analyses of exogenous $\mathrm{GnRH}$-stimulated $\mathrm{LH}$ release revealed similar secretory half-durations in older and young men, but significantly (by twofold) reduced secretory impulse amplitudes (maximal rates of bioactive $\mathrm{LH}$ secretion) in older men. There was a corresponding twofold reduction in the calculated mass of bioactive LH released per secretory peak in older men. Thus, subject to the reasonable assumption that hormone distribution volumes do not differ by this magnitude in older and young men, we can conclude that gonadotrope secretory capacity is indeed significantly reduced in healthy older men.

The exact basis for the impaired bioactive LH secretory reserve observed here in healthy aging men is not known. Diminutions in bioactive LH synthesis rates and/or incomplete posttranslational processing of $\mathrm{LH}$ in the gonadotrope can be considered (25). Such changes might result from subtle age-dependent alterations in the endogenous GnRH pulse signal, which is suggested by the broad range of physiological profiles of episodic LH secretion, as well as the occasional irregular patterns of pulsatile LH release observed in the healthy older men studied here. In addition, decreased bioactive LH secretory reserve may result from changes in the ambient sex-steroid hormone milieu $(3,26,27)$. The latter consideration is suggested by age-related alterations in plasma estradiol and/or testosterone concentrations in some, but not all, demographic studies of healthy aging male populations (7-9). In the latter regard, the present results can be formulated in relation to an overall hypothesis, in which increases in circulating estradiol concentrations with aging result in diminished bioactive LH secretion, akin to that which can be reproduced in younger men given intravenous infusions of estradiol (3); in patients with estrogen-secreting endocrine tumors (28); and in young menstruating women (29) compared with their postmenopausal counterparts $(1,29)$. Additional factors other than estrogen are also likely to be involved, since tamoxifen admin- istration did not fully reverse the observed attenuation of bioactive LH secretion in the older men. Such additional factors include-but are not limited to-illness, endogenous opiates, and/or androgens, each of which can also regulate LH bioactivity $(5,23,25)$. The role of illness can be excluded in the present studies, since the study subjects represented stable, community-based, healthy, active, aging men. The exact role(s) of other factors in age-associated decreases in bioactive LH secretory reserve will require further examination.

In conclusion, the present detailed analyses of dynamic gonadotropin release in healthy aging men have permitted us to observe significant alterations in both the quality and quantity of evoked LH release. These alterations can be shown to result from an attenuation of exogenous and endogenous GnRH-stimulated secretion of bioactive LH.

\section{Acknowledgments}

We thank Chris McNett for her skillful preparation of the manuscript; Paula P. Azimi for the artwork; the National Hormone and Pituitary Program for the provision of purified human LH; Fotini Beziriannidis for assistance in the research, and Sandra Jackson and the expert nursing staff at the University of Virginia Clinical Research Center for conduct of the research protocols.

This work was supported in part by NIH grant No. RR 00847 to the Clinical Research Center of the University of Virginia; RCDA No. 1 K04 HD 00634 (JDV): Dr. R. M. Blizzard's NIH grant AG04303-03; Diabetes and Research Training Center grant No. 5 P60 AM 2212505; and NIH-supported Clinfo Data Reduction Systems.

\section{References}

1. Dufau, M. L., J. D. Veldhuis, F. Fraioli, M. L. Johnson, and I. Beitins. 1983. Mode of secretion of bioactive luteinizing hormone in man. J. Clin. Endocrinol. \& Metab. 57:993-1000.

2. Veldhuis, J. D., M. L. Johnson, and M. L. Dufau. 1987. Preferential release of bioactive luteinizing hormone in response to endogenous and low-dose exogenous gonadotropin releasing hormone (GnRH) pulses in man. J. Clin. Endocrinol. \& Metab. 64:1275-1282.

3. Veldhuis, J. D., and M. L. Dufau. 1987. Estradiol modulates the pulsatile secretion of biologically active luteinizing hormone in man. $J$. Clin. Invest. 80:631-638.

4. Warner, B., T. J. Worgul, and L. D. Diago. 1983. Effect of very high dose d-Leu ${ }^{6}-\mathrm{GnRH}$ proethylamide on the hypothalamic-pituitary testicular axis as treatment of prostatic cancer. J. Clin. Invest. 71:1842-1853.

5. Warner, B. A., M. L. Dufau, and R. J. Santen. 1985. Effects of aging and illness on the pituitary testicular axis in men: qualitative as well as quantitative changes in luteinizing hormone. J. Clin. Endocrinol. \& Metab. 60:163-268.

6. Deslypere, J. P., J. M. Kaufman, T. Vermeulen, D. Vogelaers, J. L. Vandalem, and A. Vermeulen. 1987. Influence of age on pulsatile luteinizing hormone release and responsiveness of the gonadotropins to sex hormone feedback in men. J. Clin. Endocrinol. \& Metab. 64:68-73.

7. Zumoff, B., G. W. Strain, J. Kearns, J. O’Connor, R. S. Rosenfeld, J. Levin, and D. Fukushinum. 1982. Age variation of the 24-hour mean plasma concentrations of androgens, estrogens, and gonadotropins in normal adult men. J. Clin. Endocrinol. \& Metab. 54:534-538.

8. Davidson, J. M., J. J. Chen, L. Crapo, G. D. Gray, W. J. Greenleaf, and J. A. Catania. 1983. Hormonal changes and sexual function in aging men. J. Clin. Endocrinol. \& Metab. 57:71-77.

9. Moroz, E. V., and N. S. Verkhratsky. 1985. Hypophyseal-gonadal system during male aging. Arch. Gerontol. Geriatr. 4:13-19.

10. Baker, H. W. G., H. G. Burger, D. M. deKretser, B. Hudson, S. 
O’Connor, C. Wang, A. Mirovics, J. Court, M. Dunlop, and G. C. Rennie. 1976. Changes in the pituitary-testicular system with age. Clin. Endocrinol. 5:349.

11. Karpas, A. E., A. M. Matsumoto, C. A. Paulsen, and W. J. Bremner. 1983. Elevated serum follicle-stimulating hormone levels in men with normal seminal fluid analysis. Fertil. Steril. 39:333-336.

12. Harman, S. M., P. D. Tsitouras, P. T. Costa, and M. R. Blackman. 1982. Reproductive hormones in aging men. II. Basal pituitary gonadotropins and gonadotropin responses to luteinizing hormonereleasing hormone. J. Clin. Endocrinol. \& Metab. 54:547-552.

13. Meites, J., R. Steger, and H. Huang. 1984. Relation of neuroendocrine system to the reproductive decline in aging rats and human subjects. Fed. Proc. 39:3168-3172.

14. Karpas, A. E., W. J. Bremner, D. K. Clifton, R. A. Steiner, and D. M. Dorsa. 1983. Diminished LH pulse frequency and amplitude with aging in the male rat. Endocrinology. 112:788-792.

15. Steiner, R. A., W. J. Bremner, D. K. Clifton, and D. M. Dorsa. 1984. Reduced pulsatile $\mathrm{LH}$ and testosterone secretion with aging in the male rat. Biol. Reprod. 31:251-258.

16. Zanisi, M., E. Messi, and L. Martini. 1987. In vitro release of luteinizing hormone-releasing hormone from the hypothalamus of old male rats. Endocrinology. 120:49-54.

- 17. Dufau, M. L., R. Pock, A. Neubauer, and K. J. Catt. 1976. In vitro bioassay of $\mathrm{LH}$ in human serum: the interstitial cell testosterone (RICT) assay. J. Clin. Endocrinol. \& Metab. 42:958-964.

18. Celani, M. F., V. Montanini, G. F. Baraghimi, C. Corani, and P. Manami. 1984. Effects of acute stimulation of gonadotropin-releasing hormone $(\mathrm{GnRH})$ on biologically active serum luteinizing hormone (LH) in elderly men. J. Endocrinol. Invest. 7:589-595.

19. Veldhuis, J. D., and M. L. Johnson. 1986. Cluster analysis: a simple, versatile and robust algorithm for endocrine pulse detection. Am. J. Physiol. 250:E486-E493.

20. Veldhuis, J. D., M. L. Carlson, and M. L. Johnson. 1987. The pituitary gland secretes in bursts: appraising the nature of glandular secretory impulses by simultaneous multiple-parameter deconvolu- tion of plasma hormone concentrations. Proc. Natl. Acad. Sci. USA. 84:7686-7690.

21. Dufau, M. L., I. Z. Beitins, J. W. McArthur, and K. J. Catt. 1976. Effects of luteinizing hormone releasing hormone (LHRH) upon bioactive and immunoreactive serum LH levels in normal subjects. $J$. Clin. Endocrinol. \& Metab. 43:658-667.

22. Beitins, E. Z., M. L. Dufau, K. O'Loughlin, K. J. Catt, and J. McArthur. 1977. Analysis of biological and immunological activities in the two pools of LH released during constant infusion of LHRH in men. J. Clin. Endocrinol. \& Metab. 45:605-608.

23. Veldhuis, J. D., A. D. Rogol, M. L. Johnson, and M. L. Dufau. 1983. Endogenous opiates modulate the pulsatile secretion of biologically active luteinizing hormone in man. J. Clin. Invest. 72:20312040.

24. Veldhuis, J. D., F. Fraioli, A. D. Rogol, and M. L. Dufau. 1986. Metabolic clearance of biologically active luteinizing hormone in man. J. Clin. Invest. 77:1122-1128.

25. Dufau, M. L., and J. D. Veldhuis. 1987. Pathophysiological relationships between the biological and immunological activities of luteinizing hormone. In Balliere's Clinical Endocrinology and Metabolism. H. Burger, editor. W. B. Saunders, Philadelphia. 153-176.

26. Buckingham, J. C., and C. A. Wilson. 1985. Peripubertal changes in the nature of LH. J. Endocrinol. 104:173-177.

27. Dufau, M. L., G. D. Hodgen, A. L. Goodman, and K. J. Catt. 1977. Bioassay of circulating luteinizing hormone in the Rhesus monkey: comparison with radioimmunoassay during physiological changes. Endocrinology. 100:1557-1565.

28. Veldhuis, J. D., J. R. Sowers, A. D. Rogol, and M. L. Dufau. 1985. Pathophysiology of male hypogonadism associated with endogenous hyperestrogenism: evidence for dual defects in the gonadal axis. N. Engl. J. Med. 312:1371-1375.

29. Veldhuis, J. D., I. Z. Beitins, M. L. Johnson, M. A. Serabian, and M. L. Dufau. 1984. Biologically active luteinizing hormone is secreted in episodic pulsations that vary in relation to stage of the menstrual cycle. J. Clin. Endocrinol. \& Metab. 58:1050-1058. 